See discussions, stats, and author profiles for this publication at: https://www.researchgate.net/publication/350313034

\title{
Review Dry and Non-contact EEG Electrodes for 2010-2021 years
}

Preprint · March 2021

DOI: 10.20944/preprints202103.0555.v1

CITATIONS

0

2 authors, including:

6. Ildar Rakhmatulin

28 PUBLICATIONS 6 CITATIONS

SEE PROFILE

Some of the authors of this publication are also working on these related projects:

Project Brain computer interface View project

Project Laser weed/pest control View project 


\title{
Review Dry and Non-contact EEG Electrodes for 2010-2021 years
}

Rakhmatulin Ildar ${ }^{\mathrm{a}}$, Yajian Gan ${ }^{\mathrm{b}}$

${ }^{a}$ South Ural State University, Department of Power Plants Networks and Systems, Chelyabinsk city, Russia

${ }^{b}$ Université d'Aix-Marseille, Technopôle de Chateau Gombert, Marseille, France

Email for contact: ildar.o2010@yandex.ru

\begin{abstract}
The basis of the work of electroencephalography (EEG) is the registration of electrical impulses from the brain or some of its individual areas using a special sensor/electrode. This method is used for the treatment and diagnosis of various diseases. The use of wet electrodes in this case does not seem viable, for several well-known reasons. As a result of this, a detailed analysis of modern EEG sensors developed over the past few years is carried out, which will allow researchers to choose this type of sensor more carefully and, as a result, conduct their research more competently. Due to the absence of any standards in the production and testing of dry EEG sensors, the main moment of this manuscript is a detailed description of the necessary steps for testing a dry electrode, which will allow researchers to maximize the potential of the sensor in the various type of research.
\end{abstract}

Keywords: EEG review; dry EEG; dry EEG electrode; dry electrode; electroencephalography; non-contact electrode EEG

\section{Introduction}

Electroencephalography is a method of studying brain functions, based on the electrical impulses from their neural origins. Cohen, M. et al. [62] wrote about how electroencephalography (EEG) played an important role in the discovery of cognition, brain function, and the prospects for this direction. The main problem in reading the EEG signal by non-invasive methods is the presence of noise and artifacts. As a result, pattern recognition with EEG is often challenging due to the low signal-to-noise ratio. Aihua, Z. et al. [44] and Zhang, A. et al. [54] explained cardiac artifacts, Wiese, S. et al. [146] and Besio, W. et al. [47] examined the artifacts that occur when the electrodes were poorly connected. The influence of the environment on the electrode signal was considered by Biswas A. et al. [48], muscle artifacts considered Chen, X. et al [49] and Richer, N [50] and eye movement artifacts, the most popular artifacts, presented in papers [69]. To solve this problem, a few researchers started to process EEG signals by neural networks [70, 71, 72]. But neural networks require a lot of dataset and computing power and despite this the neural networks are increasingly being used in various fields of activity, robot control $[73,74]$ to identify various mental illnesses [51]. Over the past 
few years, the number of studies that have involved machine learning and deep learning models to recognize the EEG signal has significantly increased. For example, Amorim et al. [1], Ac1, I. et al. [2], Amin, H.. et al. [3] used machine learning to search for artifacts in the signal in the brain-computer interface (BCI) studies. Nowadays, one application is use neural networks with EEG to help the diagnosis of diseases, for example, Alzheimer's disease. The easiest way to read the EEG signal is to use dry electrodes, what demonstrated in their work Kappel, S. et al. [51] and Damalerio, R. et al. [52]. But due to many different types of electrodes, it is not easy to understand which one is better to use. For this reason, the task of this research is to analyze progress in development in EEG sensors field. For the EEG recordings, various sensors can be used: wet, dry, and non-contact. Most used wet sensors with low impedance, which usually varies within $200 \mathrm{kOhm}$ before the application of the gel and within $5 \mathrm{kOhm}$ after the application of the gel $[55,56]$. But these sensors are more suitable for laboratory research. The main disadvantage is an inconvenience. For example, setting up takes much time with wet EEG sensors. Participants need to clean the hair before starting to measure EEG. Also, the change in impedance over time due to physiological changes in the gel. At the same time, if some noise can be filtered out either by a person or by a program during the diagnosis of a disease, then in terms of machine learning, a neural network can take the slightest noise as a useful signal, which in the end will lead to a false result. Machine learning is powerful instrument but still makes mistakes, so to improve the signal-to-noise ratio of EEG, for measure EEG signal needs sensors with low noise because ECG signal is weak and sensitive to interference.

Lopez-Gordo, M. et al. [4], Molinas et al. [75], Xu et al. [76] and Chi et al. [77] - presented review dry EEG sensors. However, their study did not use a systematic approach so that the complete picture of the applications of the dry electrodes is unclear. In this paper we reviewed dry and non-contact sensors for EEG measurements. We included studies in which the test results of dry and non-contact EEG sensors presented, with impedance not more than $50 \mathrm{~K} \Omega$, since at a given impedance value it is possible to read the EEG signal [57,58]. Search for articles by keywords was carried out directly on the websites in the following publishers: Elsevier (http://www.elsevier.com/), Taylor \& Francis (https://www.tandfonline.com/), Springer (https://www.springer.com/), Wiley (https://www.wiley.com/), IEEE (https://www.iee.org/), Informa (https://www.informa.com/), MPDI (https://www.mdpi.com/), Hindawi (https://www.hindawi.com/). Keyword searches performed on the Google search engine and (https://www.researchgate.net/), (https://www.academia.edu/), Publons (https://publons.com/) mostly over the past 11 years. We used the following keywords in various combinations for the search - "dry EEG electrodes", "EEG electrodes", and others. If the results coincided, an earlier source was taken for review. The current review excludes studies focusing on the usability of electrodes and energy consumption, these points are discussed in detail in the following articles $[78,79]$. Also, we will not dwell on this theoretical moment, because Doerrfuss, J., et al. [5], Neumann, T., et al. [6], Lin, B., et al. [7], Gargiulo, G., et al. [8] described in detail the theory of using dry EEG electrodes and a description of the physical process of measuring potential. We not considered wet electrodes because since they are different in structure with dry electrodes and in last 
and there are many works on this topic $[80,81]$. Dry electrodes have several advantages and Fiedler, P., et al. [42], Kam, J., et al. [43] in their research described in detail the advantages of dry electrodes over wet electrodes. Kam, J., et al. [13] presented a comparison between dry and wet EEG. The authors showed that the performance was similar between dry and wet EEG sensors in the EEG amplitude, topography, and spectral power at rest. Dry/wet EEG electrodes might differ in other ways, for example, the standard (ANSI / AAMI), galvanostat and potentiostat, the effects of the radiation, the polarization. Since this remark is relevant to almost every work on the investigation of dry detectors, we will not mention this later every time in the analysis of the article and wrote general guidelines at the end of the manuscript.

For a clear understanding, we presented the structure of our review in fig.1.

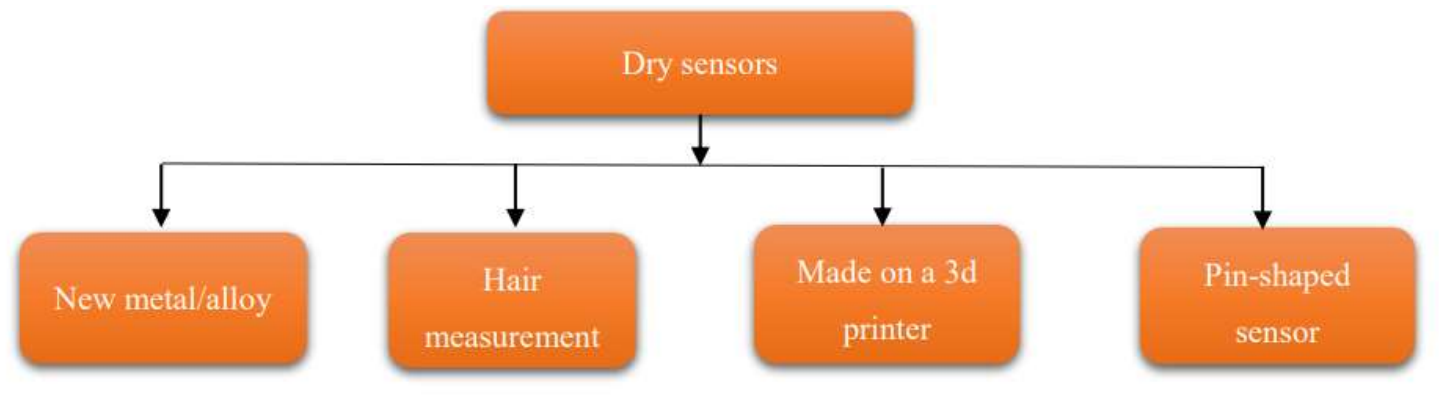

Fig. 1. Structure of review presented in the paper: new metal alloy - where new metals or their alloys were used in production, hair measurement - measurement of the EEG signal through the hair, made on a $3 \mathrm{~d}$ printer - electrodes made on a 3D printer, pin-shaped sensor - electrodes of a special shape

\section{Overview Dry EEG electrodes}

\subsection{Overview of manufacturers}

Various companies are involved in the development of electrodes for EEG. We analyzed products from the following sensor manufacturers: Cardioline (http://cardioline.com/), Fukuda Denshi (https://fukuda.com/), Edan Instruments Inc (http: // www. edan.com.cn/html/enindex.html), Biocare (https://biocare.net/), Bionet (https://www.bionetus.com/), Enobio (https://www.neuroelectrics.com /). Manufacturers that mentioned above only produce simple/basic electrodes. . To evaluate the functionality of these sensors, we introduced the following studies where authors mentioned that didn't have any conflicts of interest. Mullen et al, [35] and Mullen, T., et al. [9] presented the papers where described EEG signal in real-time and its 3D visualization with dry electrodes from Cognionics (www.cognionics.net). Qian, C., et al. [10] proposed a combined label model, including the subjective part and the objective part, to reflect the influence of the affective state in real-time. The article briefly describes the test process and focuses on the conclusions drawn in the work. Yu, M., et al. [11] and Pei, G., et al. [12] presented for registration of EEG developed Brain Power system. But in these works, only general characteristics of sensors are given, and experimental studies are not sufficiently presented. 


\subsection{Overview of new methods, ideas for the development and application of dry electrodes}

A few works with standard silver/silver chloride $(\mathrm{Ag} / \mathrm{AgCl})$ electrodes try to develop a new form of the electrode to improve the measurement [59,60], but the papers did not provide convincing evidence of the advantages of new form electrodes. Silver/silver chloride EEG electrodes are the most popular and their characteristics as impedance are well studied. Typically, this electrode is used in conjunction with a brain-computer interface, Yohanandan et al. [63], Abiri et al. [64], and Lazarou et al. [65].

Flumeri, G., et al. compared wet electrodes with three types of dry electrodes (i.e., gold-plated single pin, several pins and solid gel electrodes). Authors showed that results with dry electrodes are comparable to those with wet electrodes in terms of signal spectrum and classification of mental states. Yeon, C., et al. [15] developed a non-invasive flexible multichannel dry EEG system. This innovative mounting of the sensor using the piston-cylinder-spring assembly for attachment to the scalp. Their study focused on attaching the electrode to the scalp.

The design of active dry electrodes for BCI systems based on EEG was presented by Lee, S., et al. [16]. The electrodes consist of easy installation and high EEG quality. In this work, only special sensors are used Zhou, X., et al. presented [17] a dry electrode which low noise. The prototype of the sensor, made in a $0.18 \mu \mathrm{m}$ CMOS matrix, shows an input impedance of up to $18 \mathrm{Gm}$ at constant current and $6.7 \mathrm{Gm}$ at $50 \mathrm{~Hz}$, as well as noise at the input current of $3.03 \mu \mathrm{A} / \mathrm{VHz}$ and total input noise (IRN) $0.67 \mu \mathrm{V} \mathrm{rms}$ in the frequency band $0.5-100 \mathrm{~Hz}$. The reported characteristics of the sensor are among the best in this field. An interesting way to create an EEG electrode is to use a patterned vertical carbon nanotube (pvCNT) [18]. Electrode made on the round discs ( $\varphi=10 \mathrm{~mm}$ ) made of stainless steel. A long-term study shows minimal impedance impairment within 2 days [61]. These works $[16,17,18,61]$ are extremely difficult to reproduce as they have an individual character.

\subsection{Overview research with new alloys and materials for dry electrodes}

The physics of the process of creating electrodes by combining various alloys is described by Yu, Y. et al. [69] A few works study the possibility of using other alloys to create a dry EEG electrode besides the $\mathrm{Ag} / \mathrm{AgCl}$. [82, 83]. Camacho-Galeano E., et al. [19] analyzed in detail the characteristics of seven different materials (tin, silver, sintered $\mathrm{Ag} / \mathrm{AgCl}$, disposable $\mathrm{Ag} / \mathrm{AgCl}$, gold, platinum, and stainless steel) to produce the dry EEG electrodes. He showed that which is preferable to use gold, because line using gold electrodes does not create bias potentials. Fiedler, P., et al. [20] described a type of dry Ti / TiN electrodes. The result is that in the $\mathrm{NaCl}$ solution, the impedance of the Ti/TiN electrodes are from 824 to $54 \mathrm{Ohms}$, and the phase values are from $52^{\circ}$ to $10^{\circ}$ at frequencies from $5 \mathrm{~Hz}$ to $10 \mathrm{kHz}$, which approximates the values of the $\mathrm{Ag} / \mathrm{AgCl}$ electrodes. Mota, A ., et al. [21] presented the development of a dry electrode for recording EEG by platinum. Tests showed that the prototype of the new electrode was able to measure EEG signals better than signals of $\mathrm{Ag} / \mathrm{AgCl}$ electrodes. Liu, J., et al. [22] used a nano porous layer of platinum (Pt) that was deposited on the head of a dry electrode, reducing the contact resistance, and increasing the conductivity. The correlations between amplitude 
and latency the P300 (component of event-related potential (ERP) that manifests itself in the decision-making process) components obtained with dry and wet sensors were greater than 0.99 . Similar methods were used by Kappel, S., et al. [23], where an electrode based on a titanium (Ti) substrate coated with iridium oxide ( $\mathrm{IrO} 2)$. To sum up, the dry electrodes measurements are comparable to wet electrodes in P300 measurement. Eventually, the disadvantage of these manuscripts is the lack of lengthy tests of the developed electrodes. As a rule, research authors are limited to short tests of various types of electrodes, after which they are compared. One of the main drawbacks in the development of electrodes with new metals is the lack of polarization effect, which directly affects the potential at the electrode. Agrebi, F., et al. [24] and Yang, C. et al. [25] disclosed in detail the polarization effect.

\subsection{Overview research involving the pin-shaped dry electrodes}

A Pin-shaped electrode is the most popular form of dry electrodes. The shape is necessary for the electrodes to pass through the hair to the scalp. Because there is no fixed rule of the number of pins on the electrode and electrode, the size, the exact design of dry EEG electrodes differs between studies. Yun-Hsuan, C. et al. [26] compared and received similar result for popular forms of dry electrodes, Fig.2.

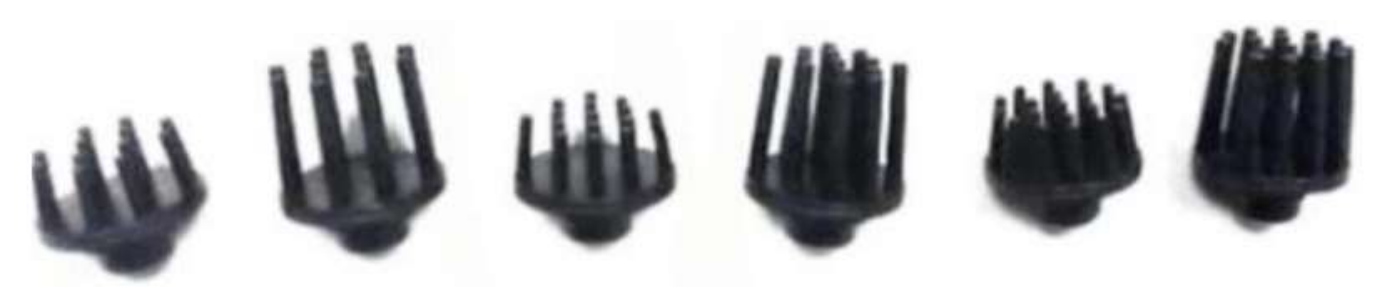

Fig.2. Pin-shaped dry sensors in various designs

Kun-Peng, G., et al. [27] developed the pin-shaped electrode with bristles for measuring the EEG signal. The pins on the dry electrode were made of carbon fibers to reduce impedance. The tips of the pins were carbon fiber bristles which could better fit the scalp. Research showed that this type of electrode is slightly inferior in signal-to-noise ratio to wet electrodes. Koctúrová, M. et al. [28], did an assessment of two types of dry EEG electrode. One was a $\mathrm{Ag} / \mathrm{AgCl}$ electrode, and the other was an electrode made of a flexible conductive polymer. Results showed that using a dry electrode with a higher resistance does not affect the measurement of the brain signal. Nathan, V., et al. [29] researched the effect of the number of pins on noise reduction. But they considered only one alloy and only one type of electrode. As a result, it is rather difficult to determine the relationship between the number of pins of pin-shaped dry sensors and the quality of the signal. Karacaoğlan, E., et al. [30] developed a silver active dry pin electrode for EEG measurements. The paper presents tests with reference to wet $\mathrm{Ag} / \mathrm{AgCl}$ electrodes, which have shown the same results. One of the generally recognized disadvantages of this type of electrode is that the pins on them very often break. This results in an impedance mismatch making the results obtained during this time interval unusable. 


\subsection{D printed dry electrodes}

Given the growing popularity of using 3D printers in various fields, researchers have tried to use 3D printing in EEG studies. Krachunov, S. et al. [31], presented a study with 3D printed dry electrodes of various shapes (fig.3). The prototypes were compared with standard wet electrodes.

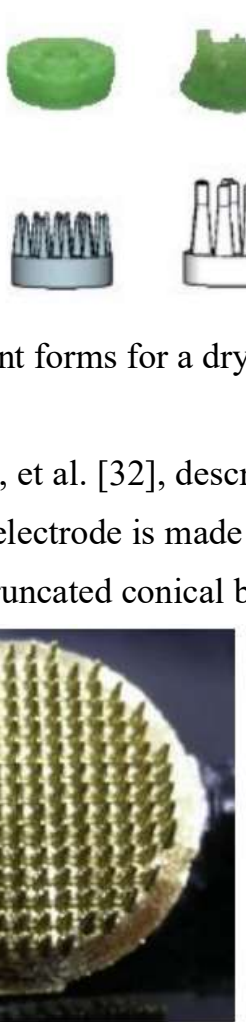

a
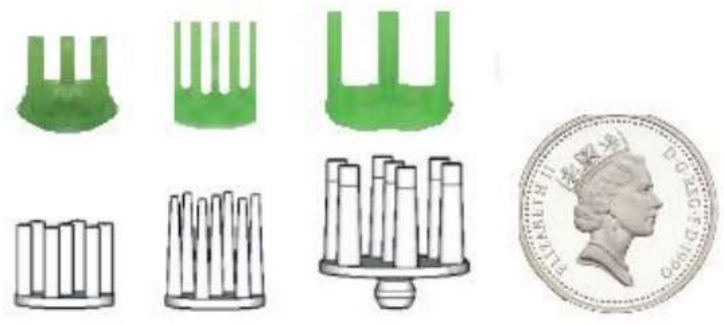

Fig. 3. Different forms for a dry electrode with a coin in denomination of one-pound sterling

Vanfleteren, J., et al. [32], described a protocol for manufacturing a dry electrode with a 3D printer. In the work, the electrode is made of an insulating acrylic-based photopolymer. It consists of 180 conical needles on a truncated conical base (fig. 4)

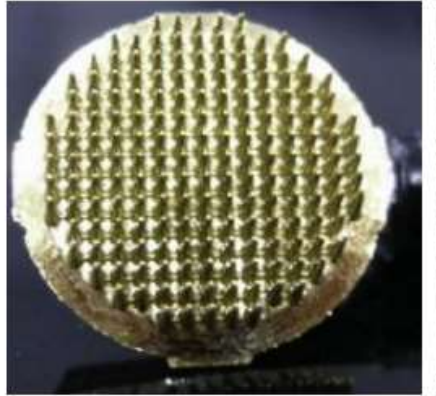

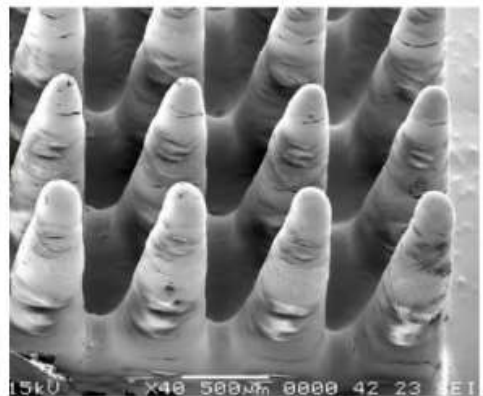

b

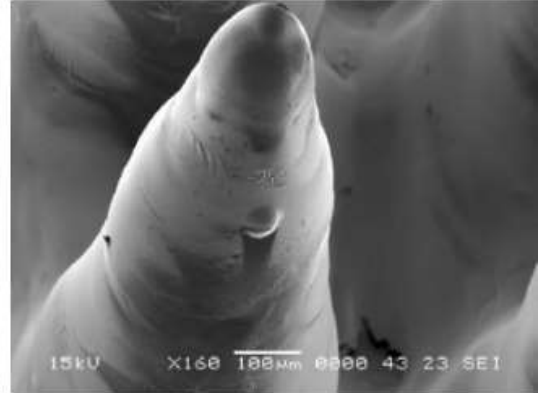

$\mathrm{c}$

Fig.4. 3D printed dry electrode: a- common view, b - Partial SEM (Scanning electron microscope) image of $\mathrm{c}$ - SEM image of a 3D printed needle tilted by $45^{\circ}$

The work also describes a metallization process consisting of two stages: sputtering of titanium as an adhesion-promoting layer and evaporation of gold to reduce impedance and prevent electrode oxidation. A comparison with wet $\mathrm{Ag} / \mathrm{AgCl}$ electrodes for recording ECG-EEG showed similar results on the quality of the signal (SNR).

Research on the use of a 3D printer is a promising direction in the manufacture of electrodes because it allows you to create the shape of the electrode, depending on the need. But the processes of titanium deposition and gold evaporation are expensive therefore it is still necessary to consider ways to reduce their cost. At the same time, there is the same problem as for electrodes made on new alloys, namely the lack of lengthy testing and investigation of the magnitude of pro-polarization.

\subsection{Overview research in which dry electrodes measure EEG in hair}

There is a limited amount of research using dry EEG through the hair (i.e., no direct contact of sensor 
and scalp). But despite this Harland et al.[33, and Oehler et al. [34] describe this direction as promising. Mullen, R. et al. [35] presented an open-source software environment for online neuroimaging and state classification and the developed EEG system with the ability to measure potential through the hair. Modeling of work yielded high accuracy (AUC $=0.97 \pm 0.021$ ) for real-time assessment of cortical connectivity. Su, L. et al. [36] developed electrodes for EEG recording in hair. Alpha rhythm and stationery visually evoked potential were tested to verify the electrode. The result suggests that the accuracy of measurement through the hair has some magnetic fields noise.

\section{Overview of non-contact sensors}

In recent years, studies using non-contact EEG is increasing, which is especially noticeable in review articles $[66,67,68]$. This is driven by the need to make the measurement process more comfortable for patients. Sullivan, T., et al. [37] developed a noise-reducing proximity EEG sensor. The sensor comes with an electronic board for amplifying and converting an analog signal to a digital signal. The electrode is in the form of a metal plate in the lower part of the printed circuit board (PCB), which is covered with a mask for soldering the noise insulation of the sensor, fig.5.

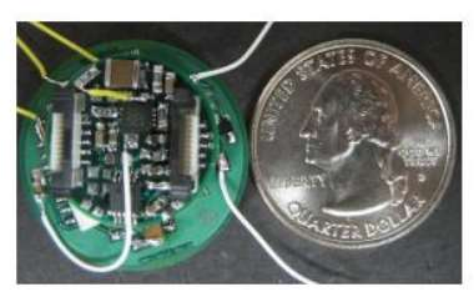

a

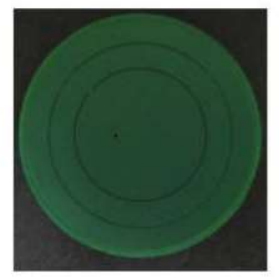

$\mathrm{b}$

Fig.5. a - two-board structure of the non-contact sensor by Sullivan et al. [37], b - The bottom side of sensor. The metal plate on the bottom is the sensor, which is covered by a solder mask

Gonzalez, S. et al. [38] proposed a new non-contact EEG sensor design. The sensor lies between the variable inter-ring distance concentric ring electrode, and a segment annular coplanar capacitive tilt sensor. This non-contact electrode improved the accuracy of the Laplacian estimation, increasing the communication ratio of the EEG, and improving artifact attenuation [fig.6].

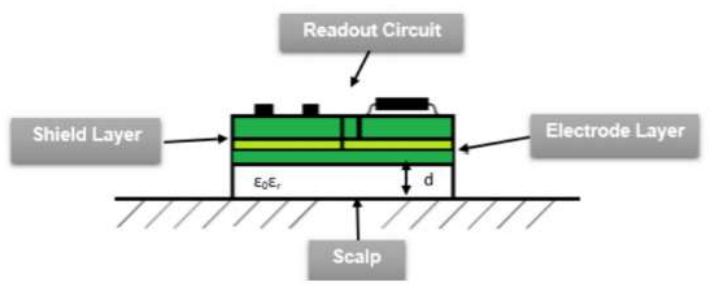

Fig. 6. PCB capacitive electrode cross section 
For the most part, the content of this work consists of a description of the technical development of the sensor. This work is more hypothetical which needs more empirical evidence to verify the effectiveness of the design. As well as Yu, M., et al. [39] who presented small non-contact EEG sensors for portable EEGs, Fig. 7.

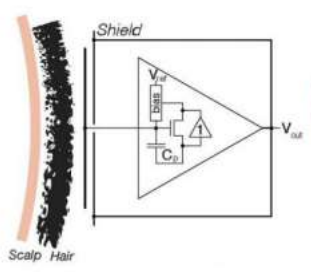

a

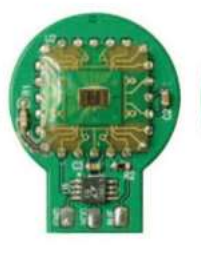

$\mathrm{b}$

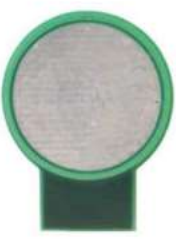

$\mathrm{c}$

Fig.7. a - integrated non-contact electrode concept, b bottom sensing plate

view from above, $\mathrm{c}$-bottom sensing plate

Data from a contactless electrode showed a maximum Information Transfer Rate ITR value of more than $19 \mathrm{bits} / \mathrm{min}$ with $100 \%$ accuracy, versus $29.2 \mathrm{bits} / \mathrm{min}$ for wet electrodes and $34.4 \mathrm{bits} / \mathrm{min}$ for dry electrodes, which is a good indicator for contactless sensors. Yu, M., et al. [40] presented wireless and contactless EEG electrodes. Each electrode provides a gain of $46 \mathrm{~dB}$ in the frequency range of $0.7-100 \mathrm{~Hz}$ with a noise level of $3.80 \mu \mathrm{V}$ to ensure the high quality of the recorded electrophysiological signals. A common disadvantage of using contactless sensors is that the amplitude levels are so low that they cannot capture short-time EEG signals. At the same time, there is high impedance with the non-contact electrodes, therefore it is necessary to use an amplifier with ultra-high impedance in the electronic circuit. Nevertheless, the above-reviewed studies still show the feasibility of using non-contact sensors in the future even they are now seemingly inferior to the contact EEG.

\section{Conclusions and discussion}

In this paper, we presented an overview of dry and non-contact EEG electrodes. We reviewed multiple works evaluating the effectiveness of their developed dry or non-contact EEG devices. To compensate for the absence of standards to judge the effectiveness of the EEG device, we recommend the following to be considered when developing and testing dry electrodes.

\section{When developing dry electrodes:}

- Need to use the electrochemical interface, such as Solartron 1287A. Using a galvanostat, we can maintain a constant current in a specific cell, regardless of the potential difference of the electrode. Using the potentiost, which is an electronic device, it is we can automatically control the potential of the electrode and maintain the set voltage value on this electrode;

- It is necessary to measure the internal noise of the electrode; 
- Before research, it is necessary to verify that the cable of electrode has immunity to frequency in the power line, immunity to radiation of radio frequencies, and immunity to radiated radio frequencies. The reduction is possible with the help of silver, graphite, and unshielded versions of screens.

\section{When testing the developed EEG:}

- Generally accepted that wet electrodes are a recognized standard, in works in which the development of a dry electrode appears, it is necessary to make a comparison with wet electrodes. In this case, it is necessary to use the standard (ANSI / AAMI) EC-12: 2000 standard for disposable electrodes. https://webstore.ansi.org/standards/aami/ansiaamiec122000r2015;

- It is necessary to use laboratory analyzers of impedance and amplitude-phase characteristics. For example, Solartron 1260 with a nominal accuracy of $0.1 \%$ from $5 \mathrm{ohms}$ to $100 \mathrm{kOhms}$. In previously reviewed manuscripts represented impedance, but there is no information on how it was measured;

- The presence of artifacts, the analysis of the obtained EEG is expediently carried out in conjunction with a neurophysiologist;

- Before testing, it is necessary to provide information about the patient's skin, resistance, and impedance;

- Only a few of the reviewed manuscript used data collection and analysis using the CorrWare software (http://www.scribner.com/software/), CorrView, ZPlot and ZView (Scribner Associates Inc. Southern Pines, NC, USA). It is advisable to use more than 1 software to ensure a more reliable result;

- Present graphs with noise voltage level. It was done in only a few works [17, 23];

- Need to refer to the standard of medical equipment (EN 60601-1-2), which describes tests for resistance to the power line frequency $(50 \mathrm{~Hz})$, magnetic field test (EN 61000-4-8), and resistance to Radio Frequency Test (RF) emissions (EN 61000-4-3). https://www.psma.com/sites/default/files/uploads/tech-forums-safety-compliance/resources/ie c60601124thedwebinarna-2.pdf $\quad$ https://standards.globalspec.com/std/1248855/EN\%2061000-4-8 https://www.emcstandards.co.uk/files/61000-4-3 immunity to radiated_rfi.pdf

We didn't find any research which fully meets the above requirements. As close as possible to a full assessment of the electrode is the thesis [41], but it is devoted to the development of a wet EEG electrode. The author recommends the necessary tests for a comprehensive check of the dry electrode. We hope this would provide a guideline for the development and performance assessment of the dry or non-contact EEG in the future. 


\section{Conflicts of Interest: None}

Funding: None

Ethical Approval: Not required

Acknowledgments: for comments, we thank Christina Y. Jin from Cognitive Modelling Group, University of Groningen

\section{References}

1. Amorim, E., Stoel, M., \& Nagaraj, S. (2019). Quantitative EEG reactivity and machine learning for prognostication in hypoxic-ischemic brain injury. Clinical Neurophysiology, 130, Issue 10, 1908-1916 2. Ac1, I., Kaya, M., \& Mishchenko, Y. (2019). Distinguishing mental attention states of humans via an EEG-based passive BCI using machine learning methods. Expert Systems with Applications, 134, 153-166

3. Amin, H., Yusoff, M., \& Ahmad, R. (2020). A novel approach based on wavelet analysis and arithmetic coding for automated detection and diagnosis of epileptic seizure in EEG signals using machine learning techniques. Biomedical Signal Processing and Control, 56, DOI: 10.1016/j.bspc.2019.101707

4. Lopez-Gordo, M.A., Sanchez-Morillo, D. \& Valle, F. (2014). Dry EEG Electrodes. Sensors, 14(7), $12847-12870$

5. Doerrfuss, J., \& Kilic, T. (2019). Predictive value of acute EEG measurements for seizures and epilepsy after stroke using a dry cap electrode EEG system - Study design and proof of concept. Epilepsy \& Behavior, 104, DOI: 10.1016/j.yebeh.2019.106486

6. Neumann, T., Baum, A., \& Baum, U. (2019). Assessment of the technical usability and efficacy of a new portable dry-electrode EEG recorder: First results of the HOMEONE study. Clinical Neurophysiology,130, Issue 11, 2076-2087.

7. Lin, B., Huang, Y., \& Lin, B. (2019). Design of smart EEG cap. Computer Methods and Programs in Biomedicine , 178, 41-46

8. Gargiulo, G., Calvo, R., Bifulco, P., \& Cesarelli, M. (2010). A new EEG recording system for passive dry electrodes. Clinical Neurophysiology ,121, Issue 5, 686-693

9. Mullen, C., Kothe1, Y., Chi, A., \& Ojeda, T. (2013). Real-Time Estimation and 3D Visualization of Source Dynamics and Connectivity Using Wearable EEG. Conference of the IEEE Engineering in Biology and Medicine Society, DOI: 10.1109/EMBC.2013.6609968

10. Qian, C., Hou, T., Lu, Y., \& Fu, S. (2018). Affective Recognition Using EEG Signal in Human-Robot Interaction. International Conference on Engineering Psychology and Cognitive Ergonomics EPCE 2018: Engineering Psychology and Cognitive Ergonomics, 336-351

11. Yu, M., Yijun, C., Wang, Y., Tzyy-Ping, J., Kerth, T., \& Cao, Y. (2013). A Practical Mobile Dry EEG System for Human Computer Interfaces. International Conference on Augmented Cognition AC, Foundations of Augmented Cognition, 649-655, DOI: 10.1007/978-3-642-39454-6_69 
12. Pei, G., Wu, J., Chen, D., Guo, G., \& Hong, M. (2018). Effects of an Integrated Neurofeedback System with Dry Electrodes: EEG Acquisition and Cognition Assessment. Sensors, 18(10), 3396, doi: 10.3390/s18103396.

13. Kam, J., Griffin, S., Shen, A., \& Patel, S. (2019). Systematic comparison between a wireless EEG system with dry electrodes and a wired EEG system with wet electrodes. NeuroImage, 184, 119-129, . doi: 10.1016/j.neuroimage.2018.09.012

14. Flumeri, G., Arico, P., Borghini, G., \& Sciaraffa, N. (2019). The Dry Revolution: Evaluation of Three Different EEG Dry Electrode Types in Terms of Signal Spectral Features, Mental States Classification and Usability. Sensors (Basel), 19(6), 1365, doi: 10.3390/s19061365

15. Yeon, C., Kim, D., Kim, K., \& Chung, E. (2014). Sensory-evoked potential using a non-invasive flexible multi-channel dry EEG electrode with vibration motor stimulation. SENSORS, 2014 IEEE, DOI: 10.1109/ICSENS.2014.6985049

16. Lee, S., Shin, Y., \& Lee, H. (2015). Design of active dry electrodes and its evaluation for EEG acquisition. International Conference on Information and Communication Technology Convergence (ICTC), DOI: 10.1109/ICTC.2015.7354609

17. Zhou, X., Li, Q., Kilsgaard, S., \& Moradi, F. (2016). A wearable ear-EEG recording system based on dry-contact active electrodes. IEEE Symposium on VLSI Circuits (VLSI-Circuits). DOI: 10.1109/VLSIC.2016.7573559

18. Abu-Saude, M., Consul-Pacareu, S., \& Morshed, B. (2015). Feasibility of patterned vertical CNT for dry electrode sensing of physiological parameters. IEEE Topical Conference on Biomedical Wireless Technologies, Networks, and Sensing Systems (BioWireleSS), DOI: 10.1109/BIOWIRELESS.2015.7152124

19. Camacho-Galeano E., Galup-Montoro C., \& Schneider, M. (2005). Evaluation of commercially available electrodes and gels for recording of slow EEG potentials. Clinical Neurophysiology, 116, 799-806, DOI:10.1016/j.clinph.2004.10.001

20. Fiedler, P., Griebel, S., Pedrosa, P., Fonseca, C., \& Vaz, F. (2015). Multichannel EEG with novel Ti/TiN dry electrodes. Sensors and Actuators A: Physical , 221, 1, 139-147

21. Mota, A., Duarte, L., Rodrigues, D., \& Martins, A. (2013). Development of a quasi-dry electrode for EEG recording. Sensors and Actuators A: Physical , 199, 1, 310-317

22. Liu, J., Liu, X., He, E., \& Gao, F. (2019). A novel dry-contact electrode for measuring electroencephalography signals. Sensors and Actuators, 294, 1, 73-80

23. Kappel, S., Rank, M., Andersen, M., \& Kidmose, P. (2019). Dry-Contact Electrode Ear-EEG. IEEE TRANSACTIONS ON BIOMEDICAL ENGINEERING, 66,1, 150-158

24. Agrebi, F., Ghorbel, N., Ladhar, A., \& Kallel, A. Enhanced dielectric properties induced by loading cellulosic nanowhiskers in natural rubber: Modeling and analysis of electrode polarization. Materials Chemistry and Physics , 200, 1, 155-163

25. Yang, C., Liu, S., \& Feng, Y. (2018). Influence of electrode polarization on the potential of DC electrical exploration. Journal of Applied Geophysics, 149, 63-76 
26. Yun-Hsuan, C., Maaike, B., \& Vanderheyden, L. (2014). Soft, Comfortable Polymer Dry Electrodes for High Quality ECG and EEG Recording. Sensors, 14, doi:10.3390/s141223758

27. Kun-Peng, G., Han-Jia, Y., Xiao-Lin, W., \& Yang, B. (2018). Soft pin-shaped dry electrode with bristles for EEG signals measurements. Sensors and Actuators A: Physical, 283, 348-361

28. Brigham, D., Singh, K., \& Pavkovic, I. (2020). Comparison of artifacts between paste and collodion method of electrode application in pediatric EEG. Clinical Neurophysiology Practice, 5, $12-15$

29. Nathan, V., \& Jafari, R. (2015). Design Principles and Dynamic Front End Reconfiguration for Low Noise EEG Acquisition with Finger Based Dry Electrodes. IEEE Transactions on Biomedical Circuits and Systems, 9, 5, 631-640

30. Karacaoğlan, E., \& Mahmut, T. (2017). The design of silver active dry with pin electrodes for EEG measurements. Medical Technologies National Congress (TIPTEKNO), DOI: 10.1109/TIPTEKNO.2017.8238048

31. Krachunov, S., \& Casson, A. (2016). 3D Printed Dry EEG Electrodes, Sensors, 16(10), 1635, doi.org/10.3390/s16101635

32. Vanfleteren, J., Schaubroeck, D., \& Raedt, R. (2012). A 3D printed dry electrode for ECG/EEG recording. Sensors and Actuators A Physical, 174(1), 96-102

33. Harland, C., Clark, T., \& Prance, R. (2002). Remote detection of human electroencephalograms using ultra high input impedance electric potential sensors. Applied Physics Letters, 81, 3284-3292 34. Oehler, M., Neumann, P., Becker, M., Curio, G., \& Schilling, M. (2008). Extraction of SSVEP signals of a capacitive EEG helmet for Human Machine Interface. 30th Annual International Conference of the IEEE Engineering in Medicine and Biology Society, DOI:10.1109/IEMBS.2008.4650211

35. Mullen, R., Kothe, A., Chi, Y., \& Ojeda, A. (2015). Real-time neuroimaging and cognitive monitoring using wearable dry EEG. IEEE Transactions on Biomedical Engineering, 99, DOI: 10.1109/TBME.2015.2481482

36. Su, L. (2015). Novel Active Comb-Shaped Dry Electrode for EEG Measurement in Hairy Site. IEEE Transactions on Biomedical Engineering, 62 , 1, 256-263

37. Sullivan, T., Deiss, S., \& Cauwenberghs, G. (2015). A Low-Noise, Non-Contact EEG/ECG Sensor, Conference: Biomedical Circuits and Systems Conference, DOI: 10.1109/BIOCAS.2007.4463332

38. Gonzalez, S. (2017). Non-Contact EEG Active Multielectrode Hardware Design. Thesis. Flinders University, 141

39. Yu, C., \& Yu-Te, W. (2011). Dry and Non-contact EEG Sensors for Mobile Brain-Computer Interfaces. IEEE transactions on neural systems and rehabilitation engineering, IEEE Engineering in Medicine and Biology Society, 20(2), 228-235

40. Yu, M., \& Gert, C. (2010). Wireless Non-contact EEG/ECG Electrodes for Body Sensor Networks. 2010 International Conference on Body Sensor Networks, DOI: 10.1109/BSN.2010.52

41. Pasi, L. (2014). Novel EEG Electrode Set for Emergency Use. Academic Dissertation. Kuopio 
University Hospital, Kuopio. Dissertations in Forestry and Natural Sciences, No 151, ISSN: 1798-5676 ISBN: 978-952-61-1551-1

42. Fiedler, P., Haueisen, J., Jannek, D., \& Griebel, S. (2014). Comparison of three types of dry electrodes for electroencephalography. ACTA IMEKO, 3, 3, 33-37, DOI: 10.21014/acta_imeko.v3i3.94

43. Kam, J., Griffin, S., Shen, A. \& Patel, S. (2019). Systematic comparison between a wireless EEG system with dry electrodes and a wired EEG system with wet electrodes. NeuroImage, 184, 119-129

44. Zhang, A., \& Weiping, L. (2004). Adaptive noise cancellation for removing cardiac and respiratory artifacts from EEG recordings, Fifth World Congress on Intelligent Control and Automation (IEEE Cat. No.04EX788), Hangzhou, China, 5557-5560, 6, doi: 10.1109/WCICA.2004.1343798

45. Zhang, A., Zheng, C., \& Jian-Wen, G. (2003). Removal of cardiac and respiratory artifacts from EEG recordings under increased intracranial pressure. Proceedings of the 2003 International Conference on Machine Learning and Cybernetics (IEEE Cat. No.03EX693), Xi'an, China, 2003, 2122-2126, 4, doi: 10.1109/ICMLC.2003.1259856

46. S. R. Wiese et al. (2005). Electrocardiographic motion artifact versus electrode impedance. IEEE Transactions on Biomedical Engineering, 52, 136-139, doi: 10.1109/TBME.2004.836503

47. Besio, W., \& Prasad, A. (2006). Analysis of Skin-Electrode Impedance Using Concentric Ring Electrode. International Conference of the IEEE Engineering in Medicine and Biology Society, New York, NY, USA, 6414-6417, doi: 10.1109/IEMBS.2006.260048

48. Biswas, D. et al. (2016). Analysing wireless EEG based functional connectivity measures with respect to change in environmental factors. IEEE-EMBS International Conference on Biomedical and Health Informatics (BHI), Las Vegas, NV, USA, 599-602, doi: 10.1109/BHI.2016.7455969

49. Chen, X., Liu,A., \& Chiang, Z. (2016). Removing Muscle Artifacts From EEG Data: Multichannel or Single-Channel Techniques?. IEEE Sensors Journal, 16, 1986-1997, doi: 10.1109/JSEN.2015.2506982

50. Richer, R. (2020). Motion and Muscle Artifact Removal Validation Using an Electrical Head Phantom, Robotic Motion Platform, and Dual Layer Mobile EEG. IEEE Transactions on Neural Systems and Rehabilitation Engineering, 28, 1825-1835, doi: 10.1109/TNSRE.2020.3000971

51. Pion-Tonachini, A., \& Makeig, S. (2018). Online Automatic Artifact Rejection using the Real-time EEG Source-mapping Toolbox (REST). 40th Annual International Conference of the IEEE Engineering in Medicine and Biology Society (EMBC), Honolulu, HI, USA, 106-109, doi: 10.1109/EMBC.2018.8512191

52. Kappel, S., Rank, M., \& Toft, H. (2019). Dry-Contact Electrode Ear-EEG. IEEE Transactions on Biomedical Engineering, 66, 150-158, doi: 10.1109/TBME.2018.2835778

53. Damalerio, R., \& Cheng, M. (2020). Development of Dry EEG Electrodes and Dry EEG Cap for Neuromonitoring. IEEE 70th Electronic Components and Technology Conference (ECTC), Orlando, FL, USA, 841-846, doi: 10.1109/ECTC32862.2020.00137 
54. Yeung, A. et al. (2015). Comparison of foam-based and spring-loaded dry EEG electrodes with wet electrodes in resting and moving conditions. 37th Annual International Conference of the IEEE Engineering in Medicine and Biology Society (EMBC), Milan, Italy, 7131-7134, doi: 10.1109/EMBC.2015.7320036

55. Fazeli, M., \& Karimi, F. (2020). Hand Motion Classification Using sEMG Signals Recorded from Dry and Wet Electrodes with Machine Learning. 2020 28th Iranian Conference on Electrical Engineering (ICEE), Tabriz, Iran, 1-4, doi: 10.1109/ICEE50131.2020.9260578

56. Yeung, A. et al. (2015). Comparison of foam-based and spring-loaded dry EEG electrodes with wet electrodes in resting and moving conditions. 37th Annual International Conference of the IEEE Engineering in Medicine and Biology Society (EMBC), Milan, Italy, 7131-7134, doi: 10.1109/EMBC.2015.7320036.

57. Fonseca, C. et al. (2007). A Novel Dry Active Electrode for EEG Recording. IEEE Transactions on Biomedical Engineering, 54, 162-165, doi: 10.1109/TBME.2006.884649

58. Nathan, V., \& Jafari, R.(2014). Reducing the noise level of EEG signal acquisition through reconfiguration of dry contact electrodes. IEEE Biomedical Circuits and Systems Conference (BioCAS) Proceedings, 572-575, doi: 10.1109/BioCAS.2014.6981790

59. Kalevo, L. et al. (2020). Effect of Sweating on Electrode-Skin Contact Impedances and Artifacts in EEG Recordings With Various Screen-Printed Ag/Agcl Electrodes. IEEE Access, 8, 50934-50943, 2020, doi: 10.1109/ACCESS.2020.2977172

60. Wu, Y., \& Chen, L. (2019). Fabrication of a PVC-based solid-state $\mathrm{Ag} / \mathrm{AgCl}$ reference electrode. IEEE International Symposium on Olfaction and Electronic Nose (ISOEN), Fukuoka, Japan, ,1-3, doi: 10.1109/ISOEN.2019.8823326

61. Debener, S. \& Bleichner, M. (2019). Transparent electroencephalography? : Exploring ear-EEG for long-term, mobile electrophysiology. 7th International Winter Conference on Brain-Computer Interface (BCI), Gangwon, Korea (South), 1-2, doi: 10.1109/IWW-BCI.2019.8737309

62. Cohen, M. (2017). Where Does EEG Come From and What Does It Mean? Trends in Neurosciences 40(4), DOI: 10.1016/j.tins.2017.02.004

63. Yohanandan, S., Kiral-Kornek, J., \& Mshford, U. (2018). A Robust Low-Cost EEG Motor Imagery-Based Brain-Computer Interface. 40th Annual International Conference of the IEEE Engineering in Medicine and Biology Society (EMBC), Honolulu, HI, USA, 5089-5092, doi: 10.1109/EMBC.2018.8513429

64. Abiri, R. et al. (2019). A comprehensive review of EEG-based brain-computer interface paradigms. Journal of Neural Engineering, 16 (1)

65. Ioulietta, L. et al. (2018). EEG-Based Brain-Computer Interfaces for Communication and Rehabilitation of People with Motor Impairment. Neuroscience, 31, https://doi.org/10.3389/fnhum.2018.00014

66. Shad, M., et al. (2020). Impedance and Noise of Passive and Active Dry EEG Electrodes: A Review. IEEE Sensors Journal, 20, 14565-14577, doi: 10.1109/JSEN.2020.3012394 
67. Xu, J., et al. (2017). Active Electrodes for Wearable EEG Acquisition: Review and Electronics Design Methodology. IEEE Reviews in Biomedical Engineering, 10, 187-198, doi: 10.1109/RBME.2017.2656388

68. Yousefi, A., \& Kassiri,H. (2020). Motion-Affected Electrode-Tissue Interface Characterization for Ambulatory EEG Recording. 42nd Annual International Conference of the IEEE Engineering in Medicine \& Biology Society (EMBC), Montreal, QC, Canada, 4479-4482, doi: 10.1109/EMBC44109.2020.9176671.

69. Ito, T., Shinji, H., Sumiya, M., \& Baba, M. Eye movement-related EEG potential pattern recognition for real-time BMI. Proceedings of SICE Annual Conference 2010, Taipei, Taiwan, 1055-1059

70. Zhang, X. Wang, W., Zhang, A., \& Chen, J. (2019). Learning Spatial-Spectral-Temporal EEG Features With Recurrent 3D Convolutional Neural Networks for Cross-Task Mental Workload Assessment. IEEE Transactions on Neural Systems and Rehabilitation Engineering, 27 (1), 31-42, doi: 10.1109/TNSRE.2018.2884641

71. Plucińska, R., et al. (2020). EEG Signal Analysis for Human Verification using Neural Networks Preliminary Experimental Results. 2020 Signal Processing Workshop (SPW), Warsaw, Poland, 6-10, doi: 10.23919/SPW49079.2020.9259137

72. Liu, Y. et al. (2016). Brain Dynamics in Predicting Driving Fatigue Using a Recurrent Self-Evolving Fuzzy Neural Network. IEEE Transactions on Neural Networks and Learning Systems, 27, 347-360, Feb. 2016, doi: 10.1109/TNNLS.2015.2496330

73. Holloman, A., \& Crawford, C. (2019). Leveraging Neurophysiological Information to Augment Interpretation of Responses to Vulnerable Robot Behaviors. 2019 14th ACM/IEEE International Conference on Human-Robot Interaction (HRI), Daegu, Korea (South), 566-567, doi: 10.1109/HRI.2019.8673290

74. Hajibabazadeh, M., \& Azimirad, V. Brain-robot interface: Distinguishing left and right hand EEG signals through SVM. 2014 Second RSI/ISM International Conference on Robotics and Mechatronics (ICRoM), Tehran, Iran, 813-816, doi: 10.1109/ICRoM.2014.6991004

75. Molinas, M., \& Ytterdal, T. Impedance and Noise of Passive and Active Dry EEG Electrodes: A Review. IEEE Sensors Journal, 20, 14565-14577, 15 Dec.15, 2020, doi: 10.1109/JSEN.2020.3012394

76. Xu, J. et al. (2017). Active Electrodes for Wearable EEG Acquisition: Review and Electronics Design Methodology. IEEE Reviews in Biomedical Engineering, 10, 187-198, 2017, doi: 10.1109/RBME.2017.2656388

77. Chi, Y., Jung, T., \& Cauwenberghs, G. (2010). Dry-Contact and Noncontact Biopotential Electrodes: Methodological Review. IEEE Reviews in Biomedical Engineering, 3, 106-119, 2010, doi: 10.1109/RBME.2010.2084078

78. Ota, S. et al. (2006). Proposal for the Extraction Method of Personal Comfort and Preference by the EEG Maps. 2006 SICE-ICASE International Joint Conference, Busan, Korea (South), 604-607, doi: 10.1109/SICE.2006.315706 
79. Amaral, V. et al. (2013). EEG signal classification in usability experiments. 2013 ISSNIP Biosignals and Biorobotics Conference: Biosignals and Robotics for Better and Safer Living (BRC), Rio de Janeiro, Brazil, 1-5, doi: 10.1109/BRC.2013.6487469

80. Lokare, N., Gonzalez, L., \& Lobaton, E. Comparing wearable devices with wet and textile electrodes for activity recognition. 2016 38th Annual International Conference of the IEEE Engineering in Medicine and Biology Society (EMBC), Orlando, FL, USA, 3539-3542, doi: 10.1109/EMBC.2016.7591492

81. Carpi, F., \& Migliorini, S. (2009). Non-invasive Wet Electrocochleography. IEEE Transactions on Biomedical Engineering, 56, 2744-2747, doi: 10.1109/TBME.2009.2026178

82. Kalevo, L. et a. (2020). Effect of Sweating on Electrode-Skin Contact Impedances and Artifacts in EEG Recordings With Various Screen-Printed Ag/Agcl Electrodes. IEEE Access, 8, 50934-50943, 2020, doi: 10.1109/ACCESS.2020.2977172

83. Molaei, S., \& Jafari, R. (2012). Study of the nonlinear behavior of the electrode-skin interface using silicon and $\mathrm{Ag} / \mathrm{AgCl}$ electrodes. 2012 IEEE International Symposium on Medical Measurements and Applications Proceedings, Budapest, Hungary, 1-5, doi: 10.1109/MeMeA.2012.6226668. 